\title{
Discrimination of Olive Oil by Cultivar, Geographical Origin and Quality Using Potentiometric Electronic Tongue Fingerprints
}

\author{
Fatma Souayah $^{1,2} \cdot$ Nuno Rodrigues $^{3,4}$ - Ana C. A. Veloso ${ }^{5,6}$ - Luís G. Dias ${ }^{1,7} \cdot$ \\ José A. Pereira $^{3} \cdot$ Souheib Oueslati $^{2} \cdot$ António M. Peres $^{8}$
}

Received: 25 July 2017 / Revised: 19 September 2017 / Accepted: 27 September 2017 / Published online: 13 October 2017

(c) AOCS 2017

\begin{abstract}
Legal regulations are set for protecting claims regarding olive oil geographical denomination. When meteorological or agroecological factors similarly affect different regions, the origin identification is a challenging task. This study demonstrated the use of a potentiometric electronic tongue coupled with linear discriminant analysis to discriminate the geographical origin of monovarietal Tunisian olive oil produced from local cv Chemlali (Kairouan, Sidi Bouzid or Sfax regions) and cv Sahli (Kairouan, Mahdia or Sousse regions). The potentiometric fingerprints of 12 or eight lipid sensors (for Chemlali and Sahli, respectively), selected using a simulated annealing meta-heuristic algorithm, allowed the
\end{abstract}

\section{António M. Peres}

peres@ipb.pt

1 Escola Superior Agrária, Instituto Politécnico de Bragança, Campus Santa Apolónia, 5300-253 Bragança, Portugal

2 Laboratoire Materiaux, Molécules et Applications (LMMA), Institut Préparatoire aux Etudes Scientifiques et Techniques (IPEST), BP 51, 2070 La Marsa, Tunisia

3 Centro de Iinvestigação de Montanha (CIMO), ESA, Instituto Politécnico de Bragança, Campus Santa Apolónia, 5300-253 Bragança, Portugal

4 Departamento de Ingeniería Agrária, Universidad de Léon, Av. Portugal, $n^{\circ}$ 41, 24071 Léon, Spain

5 Instituto Politécnico de Coimbra, ISEC, DEQB, Rua Pedro Nunes, Quinta da Nora, 3030-199 Coimbra, Portugal

6 CEB-Centre of Biological Engineering, University of Minho, Campus de Gualtar, 4710-057 Braga, Portugal

7 CQ-VR, Centro de Química-Vila Real, UTAD, Apartado 1013, 5001-801 Vila Real, Portugal

8 Laboratory of Separation and Reaction EngineeringLaboratory of Catalysis and Materials (LSRE-LCM), ESA, Instituto Politécnico de Bragança, Campus Santa Apolónia, 5300-253 Bragança, Portugal correct prediction (repeated K-fold cross-validation) of the geographic production region with sensitivities of $92 \pm 7 \%$ (Chemlali) and $97 \pm 8 \%$ (Sahli). It was also confirmed the electronic tongue capability to classify Tunisian olive oil according to olive cultivar or quality grade. The results indicated the possible use of potentiometric fingerprints as a promising innovative strategy for olive oil analysis allowing assessing geographical origin, olive cultivar and quality grade, which are key factors determining olive oil price and consumers' preference.

Keywords Electronic tongue - Chemometrics - Tunisian monovarietal olive oil · Physicochemical analysis · Sensory analysis $\cdot$ Geographical origin classification

\section{Introduction}

Olive oil quality grade classification is legally regulated by the European Union Commission and requires the assessment of physicochemical parameters and sensory attributes, envisaging the intensity perception of fruitiness as a positive attribute as well as the absence/presence of sensory defects $[1,2]$.

In addition to the official analytical methods, there is a need for simple, fast, cost-effective and environmentally friendly techniques for qualitative and/or quantitative evaluations of physicochemical and sensory sensations used for olive oil quality monitoring, traceability, authenticity and adulteration detection. Recently, Valli et al. [3] reviewed emerging novel techniques (optical spectroscopic and electronic chemical sensors-based techniques) applied, in combination with multivariate statistical models, for the establishment of useful portable instruments for in situ assessment of olive oil. The assessment of the geographical origin of 
olive oil, which is a recognized difficult task [3-5], is of utmost importance since the geographic location of the olive groves has a deep influence on the objective quality of olive oil and, so, on its commercial value. Moreover, it is a legal obligation to indicate the geographical origin in the label of olive oil sold in the European Union [6]. Several analytical methods have been used for olive oil geographical origin identification [4] some of them relying on the assessment of specific compounds (fatty acids, sterols, phenolic compounds), such as gas and liquid chromatography [7-9] and others, like mass spectrometry, nuclear magnetic resonance and spectroscopic techniques, using chemical fingerprints [4, 10-14]. The majority of these techniques requires complex and time-consuming sample pre-treatment steps, expensive equipment and skilled technicians, being not economically feasible for small olive oil producers. Thus, sensor-based electrochemical tools have emerged as potential low-cost tools for olive oil quality assessment [3]. However, the few published works that addressed the challenging task of olive oil geographical origin authentication are based on electronic noses (E-noses) or voltammetric electronic tongues (E-tongues) fingerprints [15-19], in combination with different multivariate statistical strategies (e.g., principal component analysis; linear discriminant analysis, LDA; and, artificial neural networks). In the present work, the use of a potentiometric E-tongue, comprising cross-sensitivity lipid membranes, was evaluated for the first time aiming at geographical origin assessment of Tunisian olive oil produced in geographical regions under similar climatic and agroecologic factors. For this, two monovarietal Tunisian olive oil, produced in different but close geographical regions were analyzed, namely, the predominant autochthonous Chemlali olive cultivar (samples from Kairouan, Sfax and Sidi Bouzid) and the less studied Sahli olive cultivar (samples from Kairouan, Mahdia and Sousse).

\section{Materials and Methods}

\section{Olive Oil Samples, Physicochemical and Sensory Analysis}

Sixty independent olive oil samples from 30 different commercial monovarietal olive oil were obtained from local Tunisian producers. Samples were produced in different but geographically close regions (Fig. 1) using olives from Chemlali or Sahli autochthonous varieties. From these, 34 samples were Chemlali olive oil, the predominant autochthonous olive variety, produced in Kairouan (16), Sfax (14) and Sidi Bouzid (four samples) regions. The other 26 samples of Sahli olive oil were produced in Kairouan (four), Mahdia (ten) and Sousse (12) regions. Samples were kept in the original dark amber bottles protected from the direct light

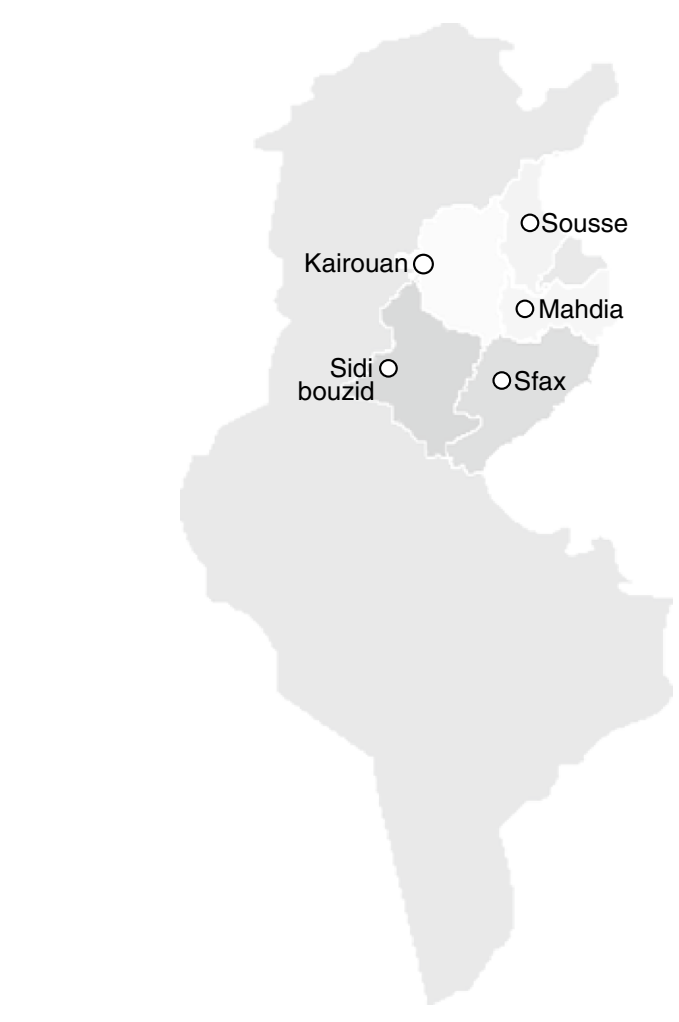

Fig. 1 Monovarietal Tunisian olive oil's geographical origin: Chemlali autochthonous olive cultivar (Kairouan, 16 samples; Sfax, 14 samples; and, Sidi Bouzid, four samples) and Sahli autochthonous olive cultivar (Kairouan, four samples; Mahdia, ten samples; and, Sousse, 12 samples)

exposition, during transport and storage. All samples were subjected to physicochemical and sensory analysis before the expiration date, following the EU regulations $[1,2]$. Free acidity (FA, in \% oleic acid), peroxide value (PV, in $\mathrm{mEq}$ $\mathrm{O}_{2} / \mathrm{kg}$ ) and the specific coefficients of extinction at 232 and $270 \mathrm{~nm}\left(K_{232}, K_{270}\right.$ and $\left.\Delta K\right)$ were evaluated by acid-base titration and UV-Vis spectrophotometry as regulated $[1,2]$. From each olive oil sample, two independent sub-samples were collected and analyzed in triplicate. The sensory evaluation followed the International Olive Council guidelines $[20,21]$ being the intensity of each attribute evaluated using a continuous scale, ranging from 0 (no intensity perceived) to 10 (maximum intensity). The intensity of positive (e.g., fruity, bitter and pungent sensations) and negative (e.g., rancid, musty, fusty and other organoleptic defects) attributes of the olive oil were assessed by eight trained panelists from a sensory panel. For each sensory evaluation, each trained panelist tasted about $15 \mathrm{~mL}$ of the olive oil served in a blue test glass, which was kept at $28 \pm 2{ }^{\circ} \mathrm{C}$ throughout the evaluation. First, the panelist smelled each sample trying to find any negative sensation as well as positive attributes, being registered as the perceived intensities in a proof sheet. Afterwards, the gustatory sensations were assessed by putting the 
sample in the mouth and with retreating breath. Regarding the negative attributes, fusty was evaluated as the characteristic flavor of olive oil produced from olives piled or that suffered advance anaerobic fermentation. Musty was assessed as the characteristic flavor of olive oil obtained from olives contaminated with fungi and yeasts due to the humid conditions during storage. Rancid, was evaluated considering the characteristic organoleptic sensation due to an intense oxidation process. Finally, the winey-vinegary attribute was classified as the characteristic reminiscent flavor of wine or vinegar perceived in the olive oil [20,21]. For the olive oil's quality grade classification, the median intensity of the defect mostly perceived was used. Also, if a negative sensation was assessed with intensity greater than 3 , the gustatory positive attributes were not evaluated. The final quality grades of all olive oil samples analyzed were established considering the physicochemical quality and the sensory data as extra-virgin olive oil (EVOO; simultaneously: FA $\leq 0.8 \%$ oleic acid, $\mathrm{PV} \leq 20 \mathrm{mEq} \mathrm{O} / 2 \mathrm{~kg}, K_{232} \leq 2.50$, $K_{270} \leq 0.22, \Delta K \leq 0.01$, median intensity of fruity positive attribute $>0$ and defects median intensities equal to 0 ), virgin olive oil (VOO; simultaneously: FA $\leq 2.0 \%$ oleic acid, $\mathrm{PV} \leq 20 \mathrm{mEq} \mathrm{O} \mathrm{O}_{2} / \mathrm{kg}, K_{232} \leq 2.60, K_{270} \leq 0.25, \Delta K \leq 0.01$, median intensity of fruity positive attribute $>0$ and median intensity of the most intense defect between 0 and 3) or lampante olive oil (LOO; other situations). All analysis were performed at the laboratories of the School of AgriculturePolytechnic Institute of Bragança (Portugal).

\section{E-tongue}

\section{E-tongue Device and Set-up}

The E-tongue device was designed and constructed as previously described $[22,23]$. The device comprised two homemade print-screen potentiometric arrays $(3 \mathrm{~cm} \times 12 \mathrm{~cm}$ visible PVC sheet plus $5 \mathrm{~cm}$ due to the RS232 plug; Fig. 2), with 40 cross-sensitivity lipid sensor membranes (diameter: $3.6 \mathrm{~mm}$; thickness: $0.3 \mathrm{~mm}$, Fig. 2). The sensor membranes (3\% of additive compound, $65 \%$ of plasticizer) resulted from different combinations of four lipid additives (octadecylamine, oleyl alcohol, methyltrioctylammonium chloride and oleic acid) and five plasticizers (bis(1-butylpentyl) adipate, dibutyl sebacate, 2-nitrophenyl-octylether, tris(2-ethylhexyl)phosphate and dioctyl phenylphosphonate) mixed with high molecular weight polyvinyl chloride $(\mathrm{PVC} ; \approx 32 \%$ ) [24]. All reagents were from Fluka (minimum purity of $97 \%$ ). For the potentiometric assays, a commercial reference $\mathrm{Ag} / \mathrm{AgCl}$ electrode was used (Metrohm Ag/ $\mathrm{AgCl}$ double junction with SGG sleeve). Each sensor was identified a letter $\mathrm{S}$ (for sensor) followed by the number of the array (1 or 2$)$ and the number of the membrane (1-20).

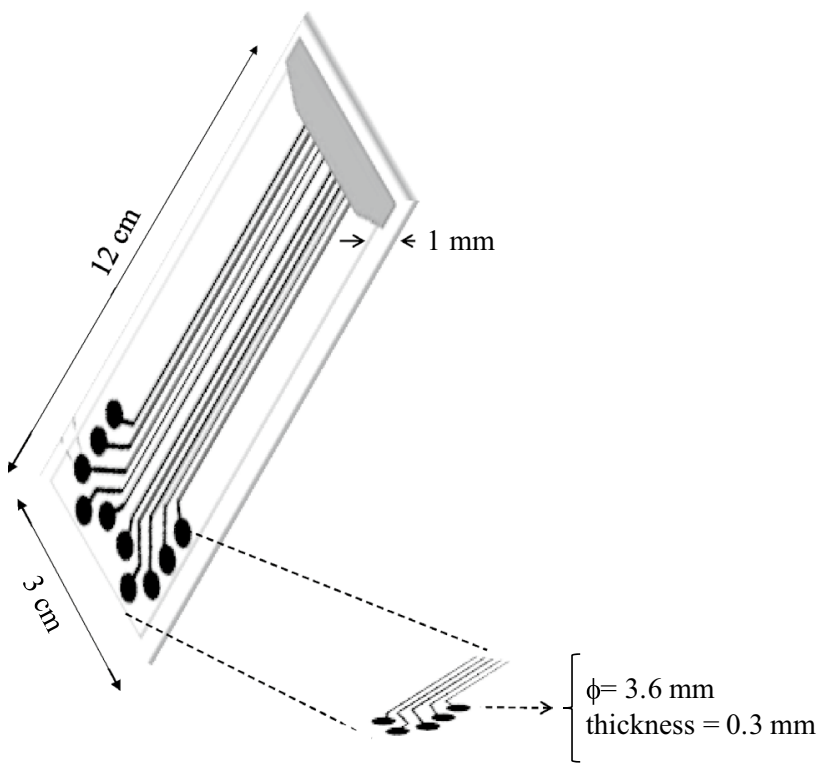

Fig. 2 E-tongue sensor screen-printed array comprising 20 lipid membranes with cross-sensitivity towards polar compounds (e.g., acids, sugars, aldehydes, esters and alcohols, etc.) related to the basic taste compounds, positive and negative sensory attributes (including, acid, sweet, bitter, pungent, umami, salty, fruity, green, rancid, wineyvinegary, etc.)

\section{E-tongue Analysis: Olive Oil Sample Preparation and Potentiometric Assays}

Olive oil ( $\approx 10 \mathrm{~g}$ ) was extracted using $100 \mathrm{~mL}$ of a water-ethanol solution $(80: 20, \mathrm{v} / \mathrm{v})$, which enabled the extraction of polar compounds related to different sensory attributes of olive oil, and to which the lipid polymeric sensors show qualitative and quantitative responses, due to electrostatic or hydrophobic interactions [22, 24-31]. Ethanol was of analytical grade (Panreac, Barcelona) and water was deionized type II. The oil-water-ethanol mixture was agitated for 5-10 $\mathrm{min}$ at 500 rpm (vortex stirrer LBX V05, lbx instruments), before standing at ambient temperature for $60 \mathrm{~min}$. After the immiscible phases separation, $40 \mathrm{~mL}$ of the supernatant solution was carefully removed and immediately analyzed with the E-tongue, for $5 \mathrm{~min}$, with retaining the potentiometric profile of the last scan, which would correspond to a pseudo-equilibrium state [24]. The analyzes were performed in duplicate, unless the coefficients of variation of the potentiometric signals recorded by each E-tongue sensor were greater than $20 \%$ [20, $21]$, in these cases a third assay was carried out. To establish the training and internal-validation datasets, there was used only one electrochemical "average" signal profile per sample [32]. Finally, since all olive oil samples were electrochemically analyzed in the same day there was no need of statistical signal pre-treatments $[22,27]$ since the potentiometric signals generated by lipid polymeric membranes usually present satisfactory intra-day (relative standard deviation percentages, RSD\%, 
lower than 20\%) $[22,27]$ and inter-days repeatabilities (RSD\% lower than 25\%) [22] as well as adequate stability in time [33].

\section{Statistical Analysis}

The statistical effect of olive cultivar (Chemali or Sahli cvs), quality grade (EVOO, VOO or LOO) or geographical location (Kairouan, Mahdia, Sidi Bouzid, Sfax or Sousse regions) on the intensities of the positive and/or negative sensory attributes of the monovarietal Tunisian olive oil, was evaluated through the $t$ Student test or the one-way analysis of variance (one-way ANOVA), followed by the Tukey's test.

The potentiometric E-tongue performance for olive oil classification according to the autochthonous Tunisian olive cultivar (i.e., cvs Chemlali or Sahli) and for each singlecultivar olive oil by its geographical origin (cv Chemlali: Kairouan, Sidi Bouzid and Sfax regions; and, cv Sahli: Kairouan, Mahdia or Sousse regions) as well as its quality grade (EVOO, VOO or LOO) was evaluated using LDA coupled with the meta-heuristic simulated annealing (SA) variable selection algorithm [34-36], following a previous described electrochemical-chemometric strategy [27-32, 37]. The sensitivities (correct classification rates) of the selected LDASA models were evaluated using the leave-one-out crossvalidation (LOO-CV) and the repeated K-fold (repeated K-fold-CV) techniques. In the latter, data was repeatedly and randomly split into $\mathrm{K}$ folds, being each of the folds left out in turn for internal-validation of the model established using the other K-1 folds [38]. In this work the number of folds was set equal to four, enabling a use of $25 \%$ of the initial data for validation purposes, with the procedure repeated ten times, turning out into 40 evaluation runs (four folds $\times$ ten repeats). The weight of each variable in the final E-tongueLDA-SA model, was normalized using variable scaling and centering procedures. The models were graphically evaluated using 1-D frequency distribution or 2-D plots. For the latter plot type, class membership boundary lines were used, being established using posterior probabilities computed through the Bayes' theorem (which enables controlling overfitting issues) to deeper assess the classification capability of the established LDA models [39]. All statistical analysis were performed using the Subselect $[35,38,40]$ and MASS [41] packages of the open source statistical program R (version 2.15.1), at a 5\% significance level.

\section{Results and Discussion}

\section{Physicochemical and Sensory Analysis of the Monovarietal Tunisian Olive Oil}

Chemlali olive oil (17 olive oil $\times$ two independent samples) and Sahli olive oil ( 13 olive oil $\times$ two independent samples) produced in different but close geographical regions, were analyzed according to the EU and IOC regulations [1, 2, $20,21]$. Physicochemical quality parameters (FA, PV, $K_{232}$, $K_{270}$ and $\Delta K$ values), the intensity of positive fruity, bitter and pungent sensations and of different negative attributes (e.g., musty, fusty, rancid) were evaluated and are shown in Table 1. Based on the physicochemical quality levels, the monovarietal olive oil could be classified as EVOO, VOO or LOO, for the two autochthonous olive cultivars (Chemlali olive oil: 28 would be classified as EVOO, four as VOO and two as LOO; Sahli olive oil: six would be classified as EVOO, two as VOO and 18 as LOO) or geographical origins. The LOO classification was mainly due to high values of $K_{232}$ followed by PV (which are positively correlated, $R$-Pearson $=+0.9370$ with a regression $P$ value $<0.001$ ), being indicators of primary oxidation of olive oil, which may be attributed to high temperatures and solar radiation in particular locations of semi-arid Tunisian regions [42]. When the sensory panel results were also taken into account for establishing the olive oil quality grade, which is legally required, the number of samples that could be classified as EVOO and VOO, based only on the physicochemical analysis, decreased substantially (Chemlali olive oil: ten EVOO, six VOO and 18 LOO; Sahli olive oil: 0 EVOO, eight VOO and 18 LOO), as shown in Table 1. This observation pointed out the need of subjecting olive oil to a sensory analysis in order to guarantee the correct quality grade classification and so to ensure the correctness of the olive oil commercial labels. Indeed, 50 out of the 60 olive oil samples evaluated were not EVOO since the panelists could perceive at least one organoleptic defect (fusty, musty, hay, brine or greasy), with rancid the defect mostly perceived. Also, for olive oil with a median intensity of the defects lower than 3 , the positive fruity sensation could be always perceived by the panelists (varying from 0.8 to 6.8 for Chemlali olive oil and from 0.5 to 7.0 for Sahli olive oil) having no statistical significant difference found between the olive cultivars ( $P$-value $=0.2691$, for $t$ Student test). In addition, for some of these olive oil samples, bitter and pungent positive sensory attributes could also be perceived but at very low intensities (varying from 0.1 to 1.7 ). It was possible to verify that Sahli olive oil possessed statistically significant higher rancidity intensities compared to Chemlali olive oil ( $P$ value $<0.0206$, for $t$ Student test). The fruity and rancid mean intensities of Chemlali olive oil did not statistically differ with the geographical origin ( $P$ value $\geq 0.0975$, for one-way ANOVA). For Sahli olive oil, the mean rancid intensities determined did not vary with the geographical origin $(P$ value $=0.0764$, for one-way ANOVA), although a statistical significant effect $(P$ value $<0.0001$, for one-way ANOVA) was found for the mean fruity intensities, showing that olive oil from Mahdia region had lower fruity intensities compared with those from Kairouan and Sousse regions 


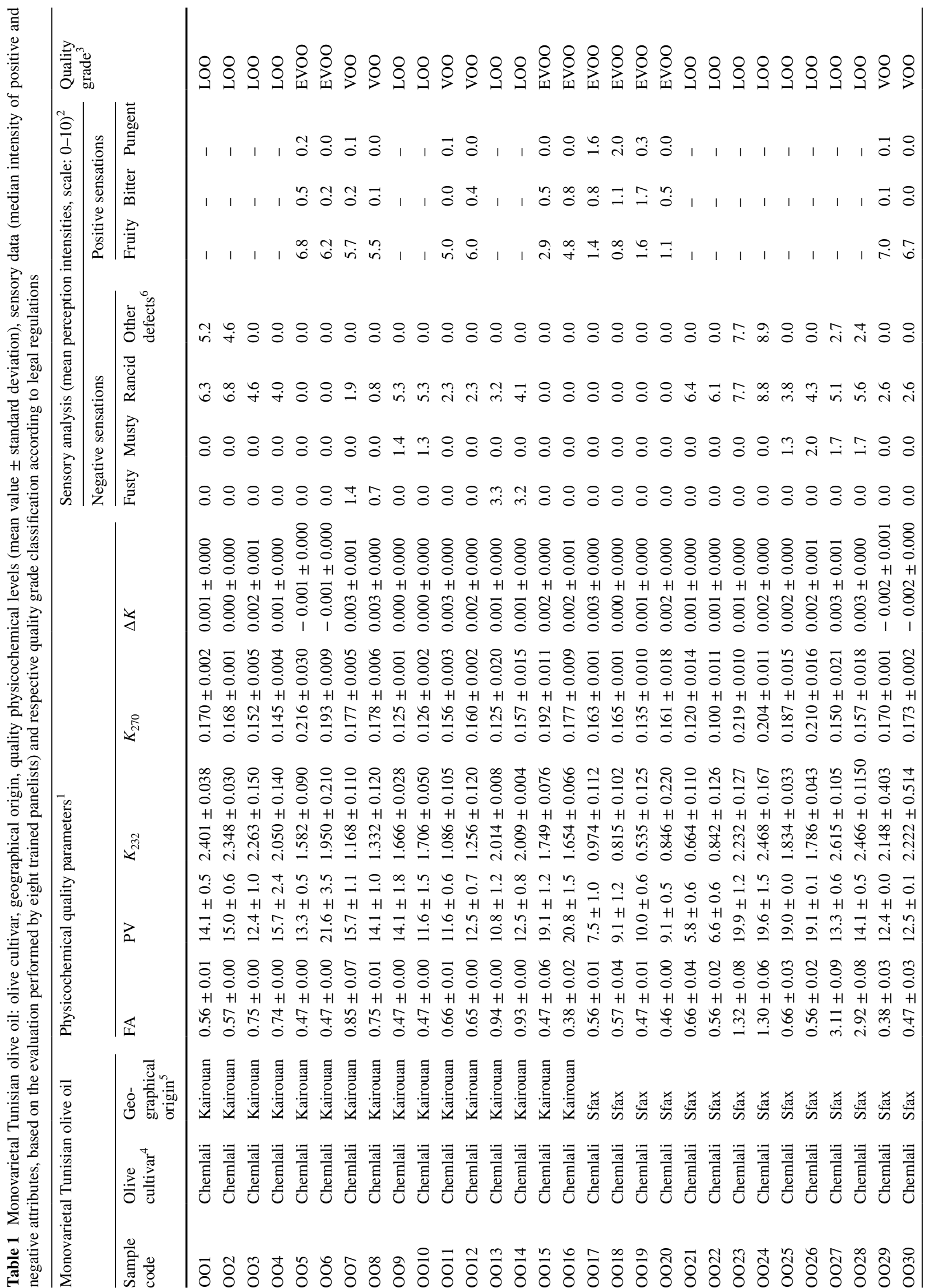




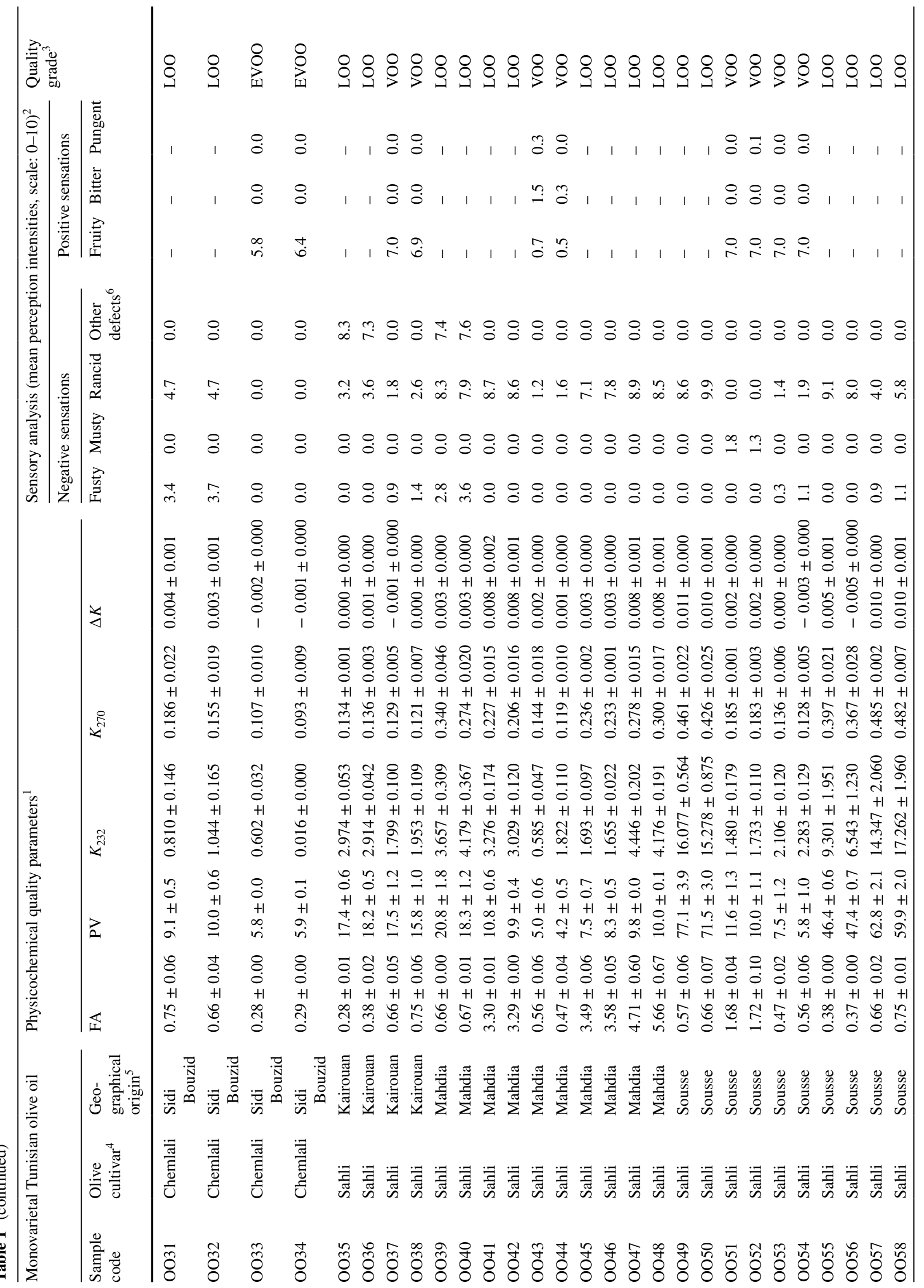




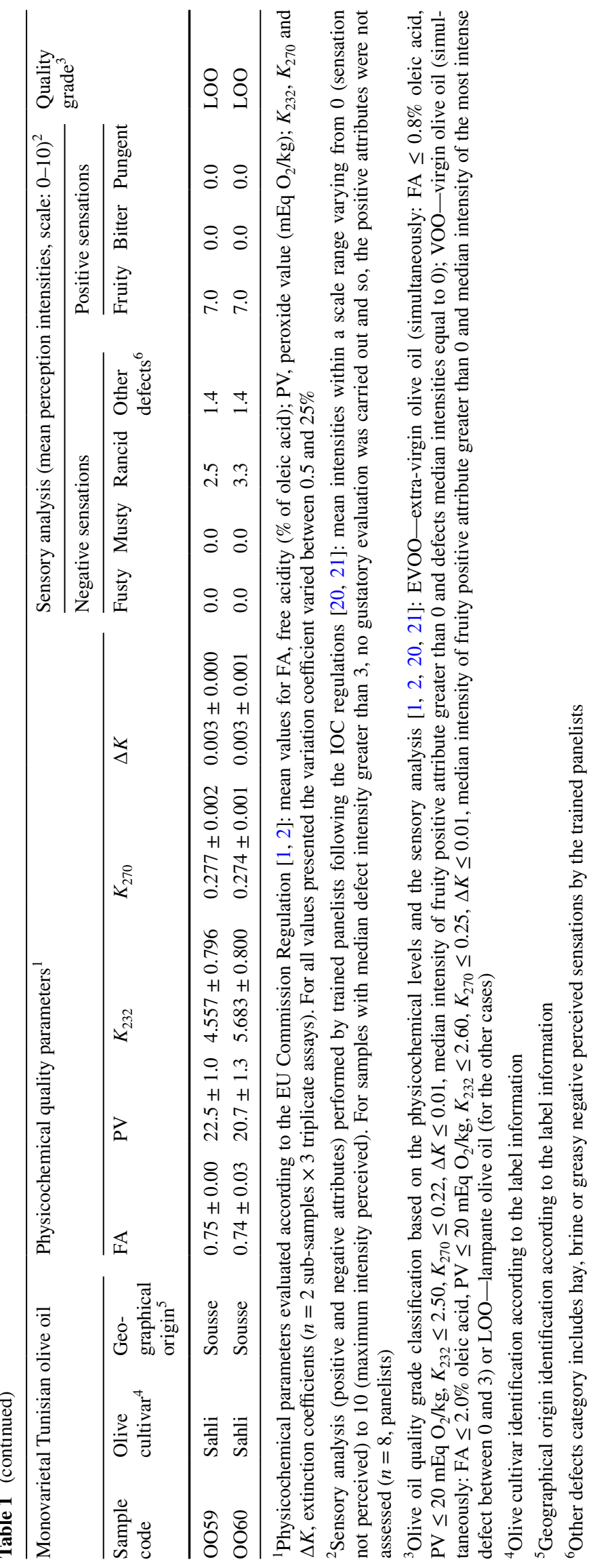


(A) Chemlali olive oil

Kairouan region

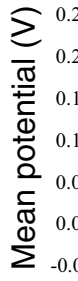

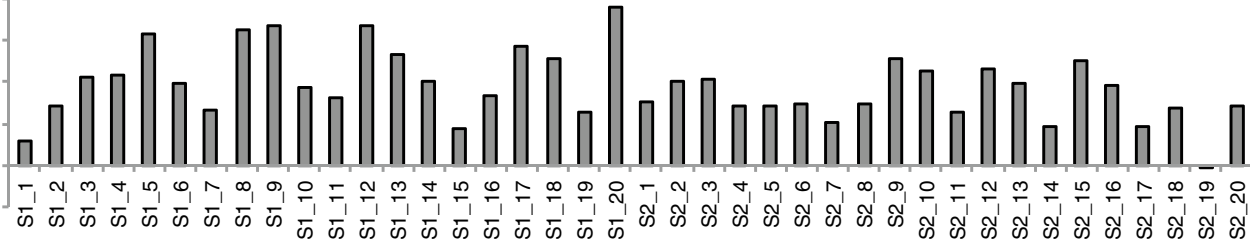

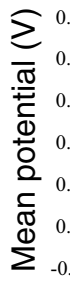

Sfax region
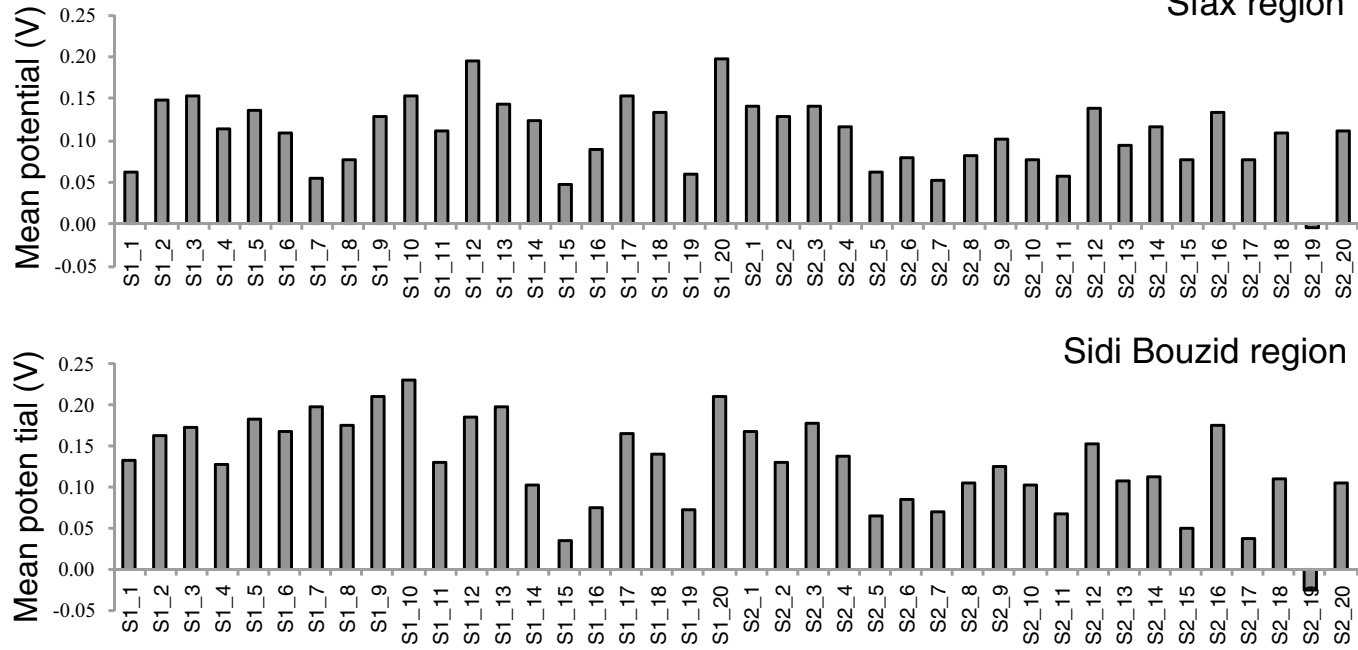

E-tongue sensor code

(B) Sahli olive oil
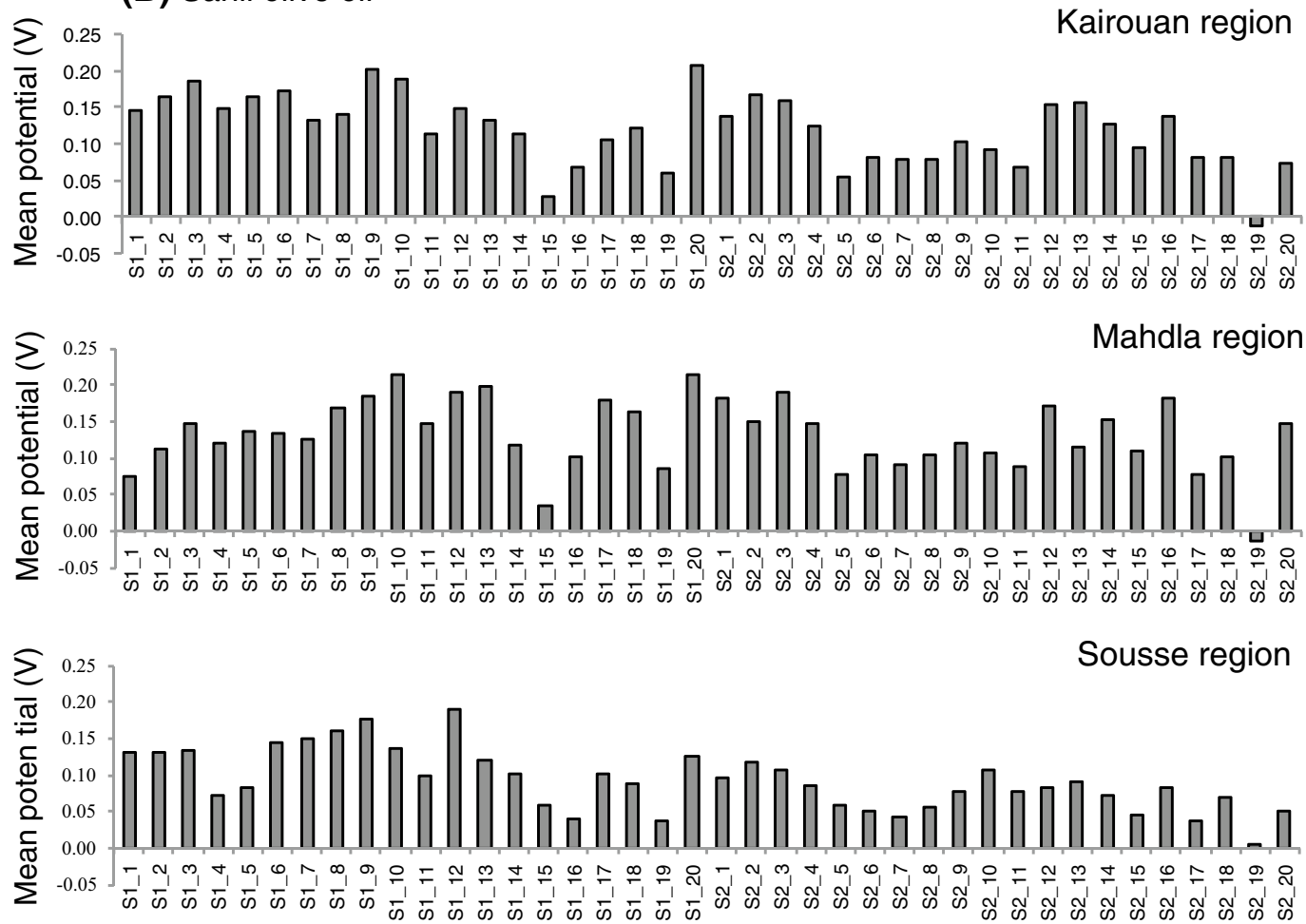

E-tongue sensor code 
4Fig. 3 Potentiometric mean signal profiles recorded by the E-tongue device during the monovarietal olive oil hydroethanolic extracts analysis (cv Chemlali or cv Sahli): overall mean signal variation with the geographical origin for each type of Tunisian autochthonous monovarietal olive oil

( $P$ value $<0.0001$, for the Tukey's test), no statistical significant difference was found between these two regions $(P$ value $=0.6453$, for the Tukey's test).

\section{Electrochemical Evaluation of Tunisian Olive Oil Using an E-tongue}

In this study it was intended to evaluate if the potentiometric E-tongue signal profiles gathered during the analysis of the hydroethanolic extracts of the monovarietal Tunisian olive oil, could be used as electrochemical fingerprints for olive cultivar differentiation, geographical origin discrimination and quality grade classification. Previously, Slim et al. [31] showed that commercial Tunisian olive oil could be satisfactorily classified according to olive cultivar (cvs. Chétoui and Sahli) and/or quality grade (EVOO, VOO and LOO) using a potentiometric device.

\section{Chemlali and Sahli Olive Oil Potentiometric Signal Profiles}

The E-tongue analysis of the olive oil hydroethanolic extracts generated potentiometric signals, varying from -307 to $+427 \mathrm{mV}$. Similarly to that previously reported by the research team $[24,31,37]$, the mean signal profiles slightly varied (data not shown) with the olive cultivar (i.e., cvs Chemlali and Sahli) and the physicochemical quality of the olive oil (i.e., EVOO, VOO or LOO). In contrast, high variability of the potentiometric typical signal profiles for each monovarietal olive oil (cvs Chemlali or Sahli) with the geographical origin was observed (Fig. 3). The signal differences (intensities and dynamic ranges) observed, may foresee feasible use for identifying the geographical origin of the studied monovarietal Tunisian olive oil.

\section{Tunisian Olive Oil Classification According to Olive Cultivar, Geographical Origin or Quality Grade, Using E-tongue Electrochemical Profiles}

In this work, E-tongue-LDA-SA models were established based on selected sub-sets of lipid sensor membranes allowing the correct classification of the 60 commercial olive oil samples, produced in different Tunisian regions (Kairouan, Mahdia, Sfax or Sousse), according to the autochthonous olive cultivar, regardless of their quality grade (EVOO, VOO or LOO). As shown in Table 2, predictive sensitivities (LOO-CV and repeated K-fold-CV) greater than $86 \%$ were obtained, which are similar to the correct classification rates (varying from 70 to $98 \%$ for LOO-CV) previously reported by Dias et al. [24, 37] for Portuguese and Spanish monovarietal olive oil and by Slim et al. [31] for Tunisian monovarietal olive oil, also using potentiometric E-tongue devices (fused or not with sensory panel data). The satisfactory performances achieved in the present study (Fig. 4a, LDA for the original grouped data) could be partially explained by the capability of potentiometric E-tongues in assessing positive (e.g., fruity) and/or negative (e.g., fusty, rancid, wetwood or winey-vinegary) attributes of olive oil [22, 26-32], which significantly differ for Chemlali and Sahli olive oil, according to the statistical analysis carried out.

From Table 2, it is also possible to verify the capability of the potentiometric E-tongue to classify samples monovarietal Tunisian olive oil according to their quality grade, confirming the results previously reported by Slim et al. [31] for Chétoui and Sahli olive oil. Indeed, it was possible to successfully classify (Fig. 4b, c, for the original grouped data) and predict the quality grade (EVOO, VOO or LOO) of Chemlali or Sahli olive oil with sensitivities greater than 83 and 99\%, respectively, which are similar to those previously described by Slim et al. [31] (varying 85 to $88 \%$ for $\mathrm{CV}$ procedures).

The use of a potentiometric E-tongue for classifying monovarietal olive oil (i.e., cvs Chemlali or Sahli) according to their geographical origin (Fig. 1) was evaluated for the first time. For Chemlali olive oil, 34 samples produced in three close but different geographical origins were used (Kairouan: 16 oils, Sfax: 14 oils; and, Sidi Bouzid: four oils) and for Sahli olive oil, 26 samples produced in other three close regions were used (Kairouan: four oils; Mahdia: ten oils; and, Sousse: 12 oils). The results (Table 2) also showed the satisfactory performance of the E-tongue-LDA-SA models established, which allowed $100 \%$ of correct classifications for the original grouped data (Fig. 5). The electrochemicalmultivariate approach used enabled achieving predictive sensitivities (for internal-validation) greater than 92 and 97\% for assessing the geographical origin of Chemlali and Sahli olive oil, respectively. Less promising results were previously described by Dias et al. [24] regarding a preliminary tentative distinction of Portuguese from Spanish olive oil (of different cultivars) using a potentiometric device. Recently, an array of voltammetric carbon paste sensors was applied to classify olive oil collected from different geographical regions of Tunisia [19]. The performance of the proposed voltammetric E-tongue was evaluated using a reduced number of olive oil (six olive oils, electrochemically analyzed several times) with different bitterness degrees, which could explained the very high correct discrimination rates reported for the original grouped data ( $\geq 98 \%)$.

The results described in the present study are very important considering that olive oil origin authentication is a 


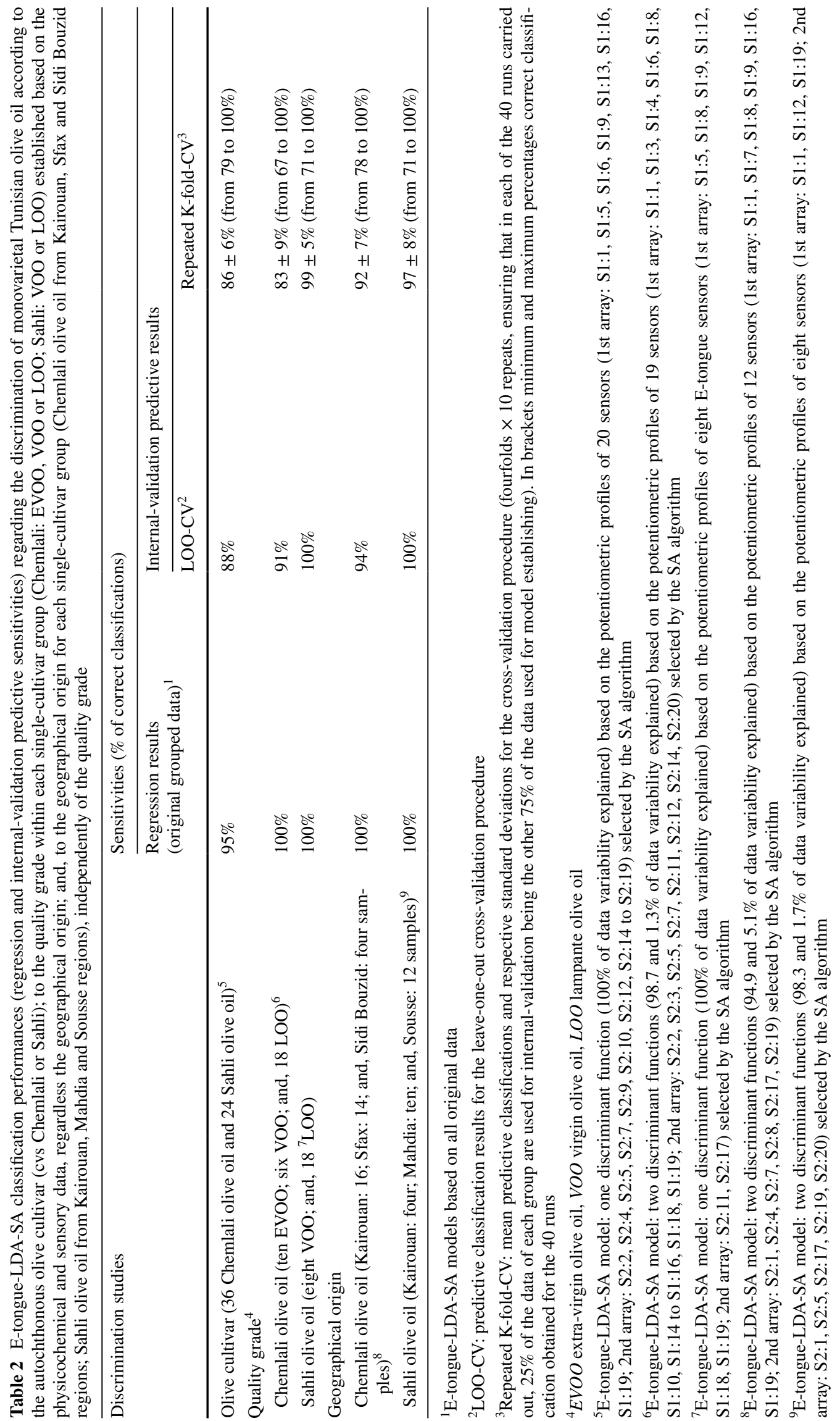


Fig. 4 Graphical representation of the E-tongue-LDA-SA performance. a Olive oil discrimination according to olive cultivar (cvs Chemlali or Sahli): classification model based on 20 selected potentiometric signals, regardless the geographical origin or quality grade. b Chemlali olive oil classification according to quality grade (EVOO, VOO or LOO): model based on 19 selected E-tongue sensors, regardless the geographical origin. The full lines represent the boundary lines based on the posterior probabilities calculated for each class membership. c Sahli olive oil classification by quality grade (VOO or LOO): model based on eight $\mathrm{E}$ selected-tongue sensors, regardless the geographical origin

challenging task namely if specific meteorological or agroecological factors influence different geographical regions similarly; a task that usually requires the combination of analytical data obtained by different analytical techniques, with multivariate approaches [4]. The satisfactory overall E-tongue predictive classification performance could be partially attributed to the fact that, in general, for the studied two single-cultivar Tunisian olive oil, different fruity and rancid intensity sensations could be perceived by the panelists depending on the geographical origin. Also, it should be remarked that the predictive geographical origin sensitivities achieved with the proposed E-tongue, used for the first time as an olive oil's origin discrimination tool, are of the same order of those obtained with E-nose systems (correct classification rates greater or equal to $96 \%$ ) $[15,17,18]$, with voltammetric E-tongue devices (correct classification rates greater or equal to $94 \%$ ) $[15,18]$ or by fusing E-nose and voltammetric E-tongue (100\% of correct classification for LOO-CV) [18].

\section{Conclusions}

Olive oil quality grades were established using both physicochemical and sensory data as legally required. The study showed the importance of the sensory evaluation of negative organoleptic attributes for the classification of olive oils as extra-virgin, virgin or lampante olive oil. It was also confirmed that the reported capabilities of the potentiometric E-tongue in combination with linear multivariate statistical tools were successful in discriminating monovarietal olive oil according to olive cultivars or commercial quality grades. This study also demonstrated that the proposed potentiometric device could be applied to classify monovarietal olive oil (with different quality grades) according to the correct geographical origin, which is legally required for olive oil labeling, for the first time to the best of the authors' knowledge. This fact is even more important, taking into account that the olive oils were produced in different but close geographical regions, influenced by similar climacteric and agroecological factors. Furthermore, it was verified that the potentiometric E-tongue showed greater sensitivities than those reported (a) Chemlali and Sahli olive oils: cultivars

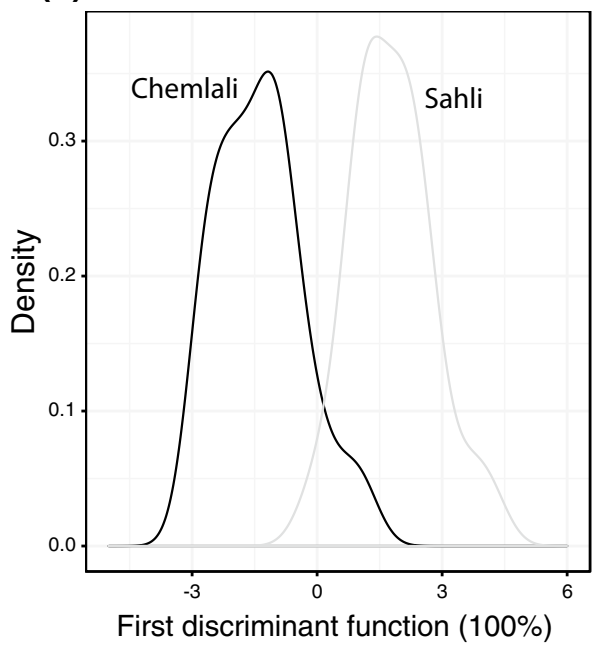

(b) Chemlali olive oils: quality grade

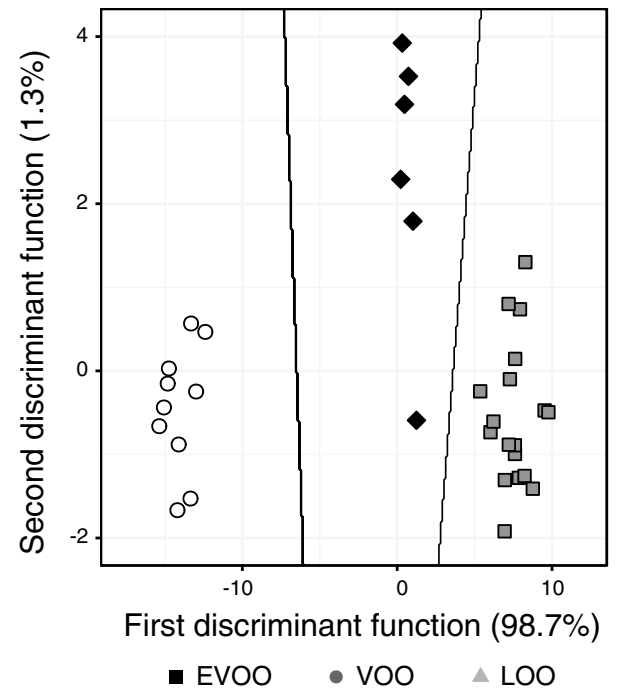

(c) Sahli olive oils: quality grade

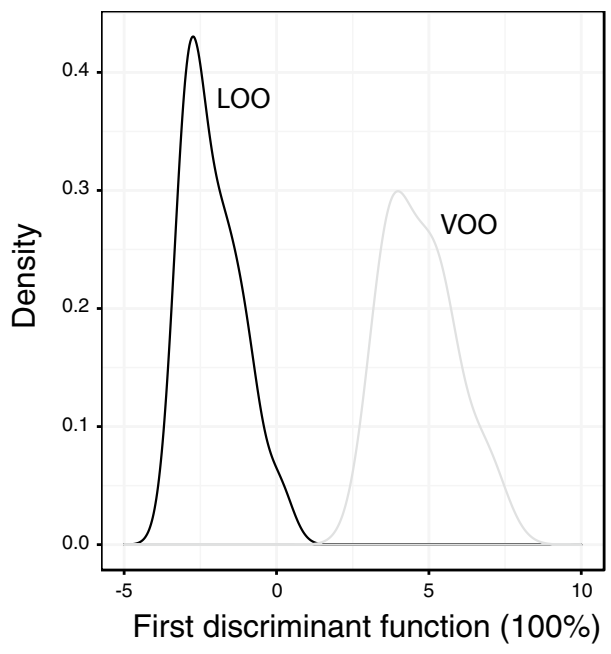


Fig. 5 Classification performance (original grouped data) of the E-tongue-LDA-SA models. a Chemlali monovarietal Tunisian olive oil discrimination (model based on 12 selected E-tongue sensors) according to geographical origin (Kairouan, Sfax, Sidi Bouzid), independently of the quality grade; b Sahli monovarietal Tunisian olive oil discrimination (model based on eight selected E-tongue sensors) according to geographical origin (Kairouan, Mahdia, Sousse), independently of the quality grade. The full lines represent the boundary lines based on the posterior probabilities calculated for each class membership (a) Chemlali olive oils: origin

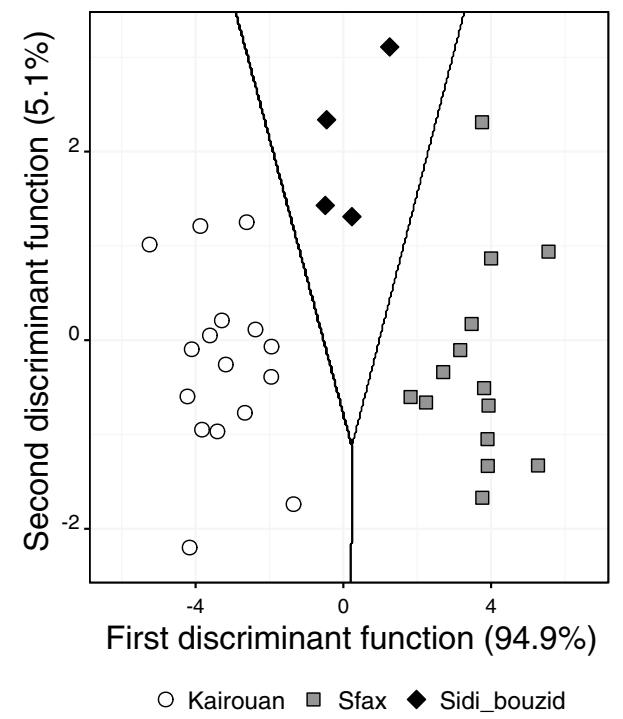

(b) Sahli olive oils: origin

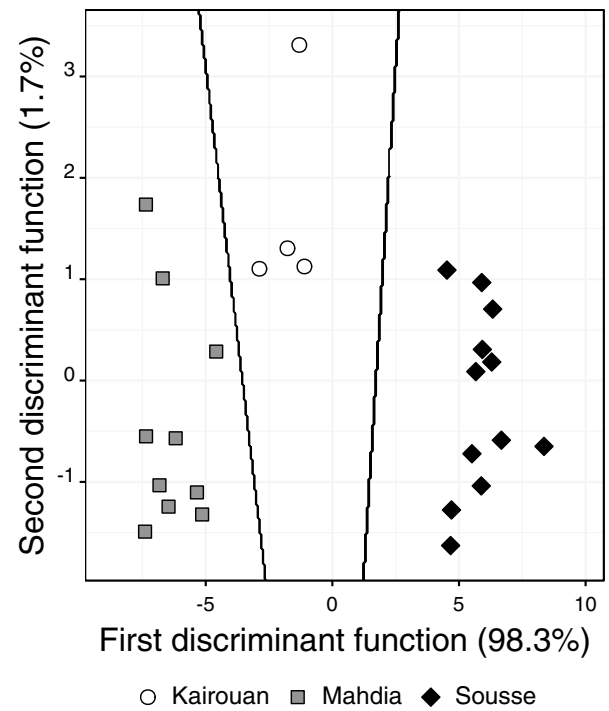

for voltammetric E-tongues and, of the same magnitude as those reported for E-nose devices, for geographical origin assessment.

Acknowledgments This work was financially supported by Project POCI-01-0145-FEDER-006984-Associate Laboratory LSRE-LCM, Project UID/QUI/00616/2013 - CQ-VR, and UID/AGR/00690/2013CIMO all funded by FEDER-Fundo Europeu de Desenvolvimento Regional through COMPETE2020-Programa Operacional Competitividade e Internacionalização (POCI) — and by national funds through FCT_Fundação para a Ciência e a Tecnologia, Portugal. Strategic funding of UID/BIO/04469/2013 unit is also acknowledged. Nuno Rodrigues thanks FCT, POPH-QREN and FSE for the Ph.D. Grant (SFRH/ $\mathrm{BD} / 104038 / 2014)$. Souheib Oueslati is also grateful for the support of the Tunisian Ministry of Agriculture.

\section{Compliance with Ethics Requirements}

Conflict of Interest The following authors have no conflict of interest: Fatma Souayah, Nuno Rodrigues, Luís G. Dias, Ana C.A. Veloso, José A. Pereira, Souheib Oueslati and António M. Peres.

Ethical Approval This article does not contain any studies with human participants or animals performed by any of the authors.

Informed Consent Not applicable.

\section{References}

1. EU No 61/2011 (2011) Commision Regulation of 24 January 2011 amending Regulation No 2568/91/EEC on the characteristics of olive oil and olive pomace oil and on the relevant methods of analysis. Off J Eur Union L23:1-13

2. EU No 1348/2013 (2013) Commission implementing regulation of 16 December 2013 amending Regulation No 2568/91/EEC on the characteristics of olive oil and olive-residue oil and on the relevant methods of analysis. Off J Eur Union L338:31-67

3. Valli E, Bendini A, Berardinelli A, Ragni L, Riccò B, Grossi M, Toschi TG (2016) Rapid and innovative instrumental approaches for quality and authenticity of olive oils. Eur J Lipid Sci Tech 118:1601-1619

4. Portarena S, Baldacchini C, Brugnoli E (2017) Geographical discrimination of extra-virgin olive oils from the Italian coasts by combining stable isotope data and carotenoid content within a multivariate analysis. Food Chem 215:1-6

5. Tena N, Wang SC, Aparicio-Ruiz R, García-González DL, Aparicio R (2015) In-depth assessment of analytical methods for olive oil purity, safety, and quality characterization. J Agric Food Chem 63:4509-4526

6. EU No 1335/2013 (2013) Commision implementing regulation of 13 December 2013 amending Implementing Regulation (EU) No 29/2012 on marketing standards for olive oil. Off J Eur Union L335:14-16

7. Aparicio R, Aparicio-Ruíz R (2000) Authentication of vegetable oils by chromatographic techniques. J Chromatogr A 881:93-104

8. Christopoulou E, Lazaraki M, Komaitis M, Kaselimis K (2004) Effectiveness of determinations of fatty acids and triglycerides for the detection of adulteration of olive oils with vegetable oils. Food Chem 84:463-474

9. Spangenberg JE, Ogrinc N (2001) Authentication of vegetable oils by bulk and molecular carbon isotope analyses with emphasis on olive oil and pumpkin seed oil. J Agric Food Chem 49:1534-1540

10. Camin F, Larcher R, Nicolini G, Bontempo L, Bertoldi D, Perini M, Schlicht C, Schellenberg A, Thomas F, Heinrich K, Voerkelius S, Horacek M, Ueckermann H, Froeschl H, Wimmer B, Heiss G, Baxter M, Rossmann A, Hoogewerff J (2010) Isotopic and elemental data for tracing the origin of European olive oils. Food Chem 58:570-577

11. Casale M, Casolino C, Oliveri P, Forina M (2010) The potential of coupling information using three analytical techniques for identifying the geographical origin of Liguria extra virgin olive oil. Food Chem 118:163-170

12. Korifi R, Le Dreau Y, Molinet J, Artaud J, Dupuy N (2011) Composition and authentication of virgin olive oil from French PDO regions by chemometric treatment of Raman spectra. J Raman Spectrosc 42:1540-1547 
13. Mannina L, Patumi M, Proietti N, Bassi D, Segre AL (2001) Geographical characterization of Italian extra virgin olive oils using high-field H-1 NMR spectroscopy. J Agric Food Chem 49:2687-2696

14. Tapp HS, Defernez M, Kemsley EK (2003) FTIR Spectroscopy and multivariate analysis can distinguish the geographic origin of extra virgin olive oils. J Agric Food Chem 51:6110-6115

15. Cosio MS, Ballabio D, Benedetti S, Gigliotti C (2006) Geographical origin and authentication of extra virgin olive oils by an electronic nose in combination with artificial neural networks. Anal Chim Acta 567:202-210

16. Guadarrama A, Rodríguez-Méndez ML, Sanz C, Ríos JL, de Saja JA (2001) Electronic nose based on conducting polymers for the quality control of the olive oil aroma. Discrimination of quality, variety of olive and geographic origin. Anal Chim Acta 432:283-292

17. Haddi Z, Amari A, Ali AO, El Bari N, Barhoumi H, Maaref A, Jaffrezic-Renault N, Bouchikhi B (2011) Discrimination and identification of geographical origin virgin olive oil by an e-nose based on MOS sensors and pattern recognition techniques. Proc Eng 2:1137-1140

18. Haddi Z, Alami H, El Bari N, Tounsi M, Barhoumi H, Maaref A, Jaffrezic-Renault N, Bouchikhi B (2013) Electronic nose and tongue combination for improved classification of Moroccan virgin olive oil profiles. Food Res Int 54:1488-1498

19. Mabrouk S, Braham Y, Barhoumi H, Maaref A (2015) Characterization and classification of different tunisian geographical olive oils using voltammetric electronic tongue. J Food Process Technol 7:534 (article 1000535)

20. IOC (2014) International Olive Council: IOC Mario Solinas quality award-Rules of the International competition for extra virgin olive oils. T.30/Doc. No 17 June 2014, p 9 (http://www.internationaloliveoil.org/)

21. IOC (2015) International Olive Council: Sensory analysis of olive oil - Method for the organoleptic assessment of virgin olive oil. COI/T.20/Doc. No 15/Rev. 8 November 2015, p 20 (http://www. internationaloliveoil.org/)

22. Veloso ACA, Silva LM, Rodrigues N, Rebello LPG, Dias LG, Pereira JA, Peres AM (2018) Perception of olive oils sensory defects using a potentiometric taste device. Talanta 176:610-618

23. Dias LG, Veloso ACA, Sousa MEBC, Estevinho L, Machado AASC, Peres AM (2015) A novel approach for honey pollen profile assessment using an electronic tongue and chemometric tools. Anal Chim Acta 900:36-45

24. Dias LG, Fernandes A, Veloso ACA, Machado AASC, Pereira JA, Peres AM (2014) Single-cultivar extra virgin olive oil classification using a potentiometric electronic tongue. Food Chem 160:321-329

25. Apetrei C, Apetrei IM, Villanueva S, de Saja JA, GutierrezRosales F, Rodriguez-Mendez ML (2010) Combination of an e-nose, an e-tongue and an e-eye for the characterisation of olive oils with different degree of bitterness. Anal Chim Acta 663:91-97

26. Kobayashi Y, Habara M, Ikezazki H, Chen R, Naito Y, Toko K (2010) Advanced taste sensors based on artificial lipids with global selectivity to basic taste qualities and high correlation to sensory scores. Sensors 10:3411-3443

27. Marx IMG, Rodrigues N, Dias LG, Veloso ACA, Pereira JA, Drunkler DA, Peres AM (2017) Quantification of table olives' acid, bitter and salty tastes using potentiometric electronic tongue fingerprints. LWT Food Sci Technol 79:394-401

28. Marx IMG, Rodrigues N, Dias LG, Veloso ACA, Pereira JA, Drunkler DA, Peres AM (2017) Assessment of table olives' organoleptic defects intensities based on the potentiometric fingerprint recorded by an electronic tongue. Food Bioprocess Tech 10:1310-1323

29. Marx Í, Rodrigues N, Dias LG, Veloso ACA, Pereira JA, Drunkler DA, Peres AM (2017) Sensory classification of table olives using an electronic tongue: analysis of aqueous pastes and brines. Talanta 162:98-106

30. Veloso ACA, Dias LG, Rodrigues N, Pereira JA, Peres AM (2016) Sensory intensity assessment of olive oils using an electronic tongue. Talanta 146:585-593

31. Slim S, Rodrigues N, Dias LG, Veloso ACA, Pereira JA, Oueslati S, Peres AM (2017) Application of an electronic tongue for Tunisian olive oils' classification according to olive cultivar or physicochemical parameters. Eur Food Res Technol 243:1459-1470

32. Rodrigues N, Dias LG, Veloso ACA, Pereira JA, Peres AM (2016) Monitoring olive oils quality and oxidative resistance during storage using an electronic tongue. LWT Food Sci Technol 73:683-692

33. Dias LG, Peres AM, Veloso ACA, Reis FS, Vilas Boas M, Machado AASC (2009) An electronic tongue taste evaluation: identification goat milk adulterations with bovine milk. Sens. Actuator B Chem 136:209-217

34. Bertsimas D, Tsitsiklis J (1992) Simulated annealing. Stat Sci 8:10-15

35. Cadima J, Cerdeira JO, Minhoto M (2004) Computational aspects of algorithms for variable selection in the context of principal components. Comput Stat Data Anal 47:225-236

36. Kirkpatrick S, Gelatt CD, Vecchi MP (1983) Optimization by simulated annealing. Science 220:671-680

37. Dias LG, Rodrigues N, Veloso ACA, Pereira JA, Peres AM (2016) Monovarietal extra virgin olive oils classification: a fusion of human sensory attributes and an electronic tongue. Eur Food Res Technol 242:259-270

38. Kuhn M, Johnson K (2013) Applied predictive modeling. Springer Science Business Media, New York

39. Bishop CM (2006) Pattern recognition and machine learning, 1st edn. Springer, New York

40. Cadima J, Cerdeira JO, Silva PD, Minhoto M (2012) The subselect R package (http://cran.rproject.org/web/packages/subselect/ vignettes/subselect.pdf.\%20Accessed\%2015/02/2016)

41. Venables WN, Ripley BD (2002) Modern applied statistics with $\mathrm{S}$ (statistics and computing), 4th edn. Springer, New York

42. Loubiri A, Taamalli A, Talhaoui N, Mohamed SN, Carretero AS, Zarrouk M (2017) Usefulness of phenolic profile in the classification of extra virgin olive oils from autochthonous and introduced cultivars in Tunisia. Eur Food Res Technol 243:467-479 